\title{
Automatic Classification of Superimposed Modulations for 5G MIMO Two-Way Cognitive Relay Networks
}

\author{
Haithem Ben Chikha and Ahmad Almadhor \\ Jouf University, College of Computer and Information Sciences, Computer Engineering and Networks Department, \\ Sakaka, 72388, Kingdom of Saudi Arabia \\ *Corresponding Author: Ahmad Almadhor. Email: aaalmadhor@ju.edu.sa \\ Received: 22 March 2021; Accepted: 09 June 2021
}

\begin{abstract}
To promote reliable and secure communications in the cognitive radio network, the automatic modulation classification algorithms have been mainly proposed to estimate a single modulation. In this paper, we address the classification of superimposed modulations dedicated to $5 \mathrm{G}$ multipleinput multiple-output (MIMO) two-way cognitive relay network in realistic channels modeled with Nakagami-m distribution. Our purpose consists of classifying pairs of users modulations from superimposed signals. To achieve this goal, we apply the higher-order statistics in conjunction with the MultiBoost AB classifier. We use several efficiency metrics including the true positive (TP) rate, false positive (FP) rate, precision, recall, F-Measure and receiver operating characteristic (ROC) area in order to evaluate the performance of the proposed algorithm in terms of correct superimposed modulations classification. Computer simulations prove that our proposal allows obtaining a good probability of classification for ten superimposed modulations at a low signal-to-noise ratio, including the worst case (i.e., $\mathrm{m}=0.5$ ), where the fading distribution follows a one-sided Gaussian distribution. We also carry out a comparative study between our proposal using MultiBoostAB classifier with the decision tree (J48) classifier. Simulation results show that the performance of MultiBoostAB on the superimposed modulations classifications outperforms the one of J48 classifier. In addition, we study the impact of the symbols number, path loss exponent and relay position on the performance of the proposed automatic classification superimposed modulations in terms of probability of correct classification.
\end{abstract}

Keywords: Automatic classification; MIMO two-way cognitive relay network; Nakagami- $m$ channels; superimposed modulations; $5 \mathrm{G}$

\section{Introduction}

Recently, a lot of attention has been paid to the two-way relaying (TWR) scheme, which consists of the exchange information between two users via a commonly shared relay in the absence of a direct link between them [1-6]. The transmission process under a TWR channel (TWRC) is performed in two-time slots. In the first time slot, the two user nodes send signals 
to the relay node. In the second time slot, the relay node broadcasts the received signals to the users. In this context, the physical-layer network coding (PNC) introduced in [7], is proposed to allow the relay node to decode a linear function of the received signals, and thereafter to allow each user to decode the incoming message from the other user based on the self-message. The PNC can double the throughput of a TWRC compared to the conventional one-way relay channel by decreasing the time slots for the exchange of one packet from four to two $[7,8]$. It can acheive $1 / 2$ bit of the capacity using a single-input single-output (SISO) Gaussian TWRC and it is assymptotically optimal in the presence of high signal-to-noise ratio (SNR) levels [9]. Note that concurrent transmissions based on PNC provide a high spectrum efficiency compared to the network coding and time-division solutions [10]. Consequently, the TWR finds applications in a wide range of applications envisaged for 5 th generation new radio (5G NR) wireless networks and beyond, including the streaming $4 \mathrm{~K}$ video, on-line cloud sharing and machine-to-machine communications [11,12]. For the purpose of obtaining a good performance when using PNC for these applications, various studies have been carried out with a specific focus on TWRC issues, including the design of symbol mapping [13,14], channel estimation [15,16] and phase synchronization or effect of time [17-20]. For satellite communication applications, a satellite can serve as a relay to enable the simultaneous information exchange between two ground stations. In this context, it is necessary to design heterogeneous modulation PNC to allow the exchange unequal amount of data between the two ground stations and/or to exchange data in asymmetric channel conditions of the two ground station-satellite links. Hence, each station may vary the used modulation type and order. Thus, each station needs the knowledge of the modulation scheme employed by the other station in order to properly demodulate the incoming signal. To guarantee an accurate data reception at the stations, a correct detection of the stations modulations from the superimposed symbols is demanded. It is well-known that multiple-input multiple-output (MIMO) system can offer a considerable gain compared to SISO system, especially in environments presenting rich-scattering. In fact, it is a popular technique for increasing the spectral efficiency and the reliability of cellular networks. For that reason, deploying many antenna elements in MIMO systems is one of the most promising technologies in $5 \mathrm{G}$ NR systems that can enable beamforming and spatial multiplexing [21]. In a MIMO receiver node, the space-time decoder or the spatial demultiplexer and the demodulator are used to recover the transmitted binary information. In fact, the receiver node is the entity that converts the received waves into a binary stream. Consequently, the estimation of the transmitted binary information necessitates a prior knowledge of the communication parameters, such as the number of the source antennas, coding, noise variance, channel matrix, and modulation. To design efficient cognitive radio, several algorithms dedicated to the estimation of the communication parameters have been proposed in the literature. The authors in [22-24], have proposed algorithms for estimating the number of the source antennas. On the other hand, many approaches have been proposed for the detection of the coding $[25,26]$. Other algorithms dedicated to the channel matrix estimation are available in [27,28]. The most widely existing modulation classification algorithms in the literature are proposed to estimate a single M-ary modulation [29-34]. However, the superposition of two M-ary modulations leads to a significant augmentation of the resulting constellation size in addition to an unusual spatial arrangement [35,36]. In fact, the superposition of two modulations with orders $\mathbf{M}_{1}$ and $\mathbf{M}_{2}$ leads to a modulation with an order upper bounded by $\mathrm{M}_{1} \times \mathrm{M}_{2}$. Furthermore, the superimposed modulated useful information with the noise results in the dispersion of the constellation points from their appropriate positions. In [35,36], the authors have addressed the problem of modulation classification of superimposed modulations in two-way relaying MIMO systems with PNC under Rayleigh channels. In addition, the zero-forcing $(\mathrm{ZF})$ precoding technique is applied at each source 
node before transmission. To the best of the authors' knowledge, there is no previous work, which focused on the problem of superimposed modulations classification for MIMO two-way cognitive relay (TWCR) network under realistic channel modeled by Nakagami-m.

In this paper, we propose an algorithm dedicated to the classification of the superimposed users modulations for MIMO TWCR network under Nakagami-m channels. Here, we use a ZF processing at the relay node. The purpose is to classify pairs of users modulations for given superimposed constellations in the case of the presence of Nakagami-m fading. At the relay node, we extract the higher-order statistics (HOSs) of the equalized superimposed symbols as an input to the MultiBoostAB classifier. To evaluate the performance of this latter classifier in terms of modulation classification, several efficiency metrics, such as the true positive rate, falsepositive rate, precision, recall, F-Measure and receiver operating characteristic (ROC) area are used. Simulation results illustrate that the proposed algorithm can achieve a good classification probability of modulations pair even in the worst case of Nakagami-m (i.e., $\mathrm{m}=0.5$ ) at a low signal-to-noise ratio (SNR).

The rest of this paper is organized as follows: In Section 2, we model the considered MIMO TWCR network. In Section 3, we describe the proposed modulation classification algorithm. Our main results are illustrated in Section 4. Section 5 gives the conclusion of the paper.

Mathematical Notations: $E[$.$] stands for expectation. (\cdot)^{-1}$ and $(\cdot)^{H}$ denote the inverse and the conjugate transpose operations, respectively. $\mathbb{C}^{L \times C}$ represents the set of $L \times C$ matrices over complex field.

\section{Considered MIMO TWCR Network}

A MIMO TWCR network is considered as shown in Fig. 1, where two users denoted as $\mathrm{U}_{1}$ and $\mathrm{U}_{2}$ and equipped with $\mathrm{N}_{\mathrm{U}_{1}}$ and $\mathrm{N}_{\mathrm{U}_{2}}$ antennas, respectively, exchange information through a common relay $(\mathrm{R})$ equipped with $\mathrm{N}_{\mathrm{R}}$ antennas.

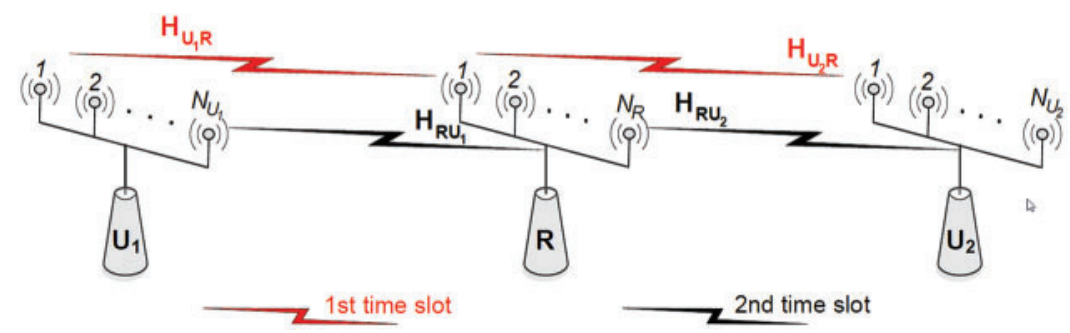

Figure 1: MIMO TWRC network

For simplicity, an equal $\mathrm{N}_{\mathrm{U}}$ number of antennas is assumed at all nodes (i.e., $\mathrm{N}_{\mathrm{U}}=\mathrm{N}_{\mathrm{U}_{1}}=$ $\mathrm{N}_{\mathrm{U}_{2}}=\mathrm{N}_{\mathrm{R}}$ ). Here, we consider that there is no direct link between $\mathrm{U}_{1}$ and $\mathrm{U}_{2}$ due to the presence of a heavy shadowing. In our transmission model, the message exchange takes place in two time slots. In the first time slot, a multiple access (MAC) phase, in which both users $\mathrm{U}_{1}$ and $\mathrm{U}_{2}$, simultaneously send their signals to $R$, is performed. Here, we consider that $U_{1}$ and $U_{2}$ use two modulations with orders $\mathrm{M}_{1}$ and $\mathrm{M}_{2}$. Their superposition leads to a modulation with an order upper bounded by $\mathrm{M}_{1} \times \mathrm{M}_{2}$. We consider ten combinations of modulation pairs reported in Tab. 1. 
Table 1: Considered combinations of modulation pairs

\begin{tabular}{ll}
\hline Index & $\mathcal{M}_{i}$ \\
\hline 1 & $($ 16QAM, 16QAM) \\
2 & $($ 16QAM, 64QAM) \\
3 & (16QAM, 2PSK) \\
4 & (16QAM, 4PSK) \\
5 & (64QAM, 64QAM) \\
6 & (64QAM, 2PSK) \\
7 & (64QAM, 4PSK) \\
8 & (2PSK 2PSK) \\
9 & (2PSK, 4PSK) \\
10 & (4PSK, 4PSK) \\
\hline
\end{tabular}

For example, in Fig. 2, we show the constellation of the superposition of a 4PSK and a 16QAM that contains 64 different points.

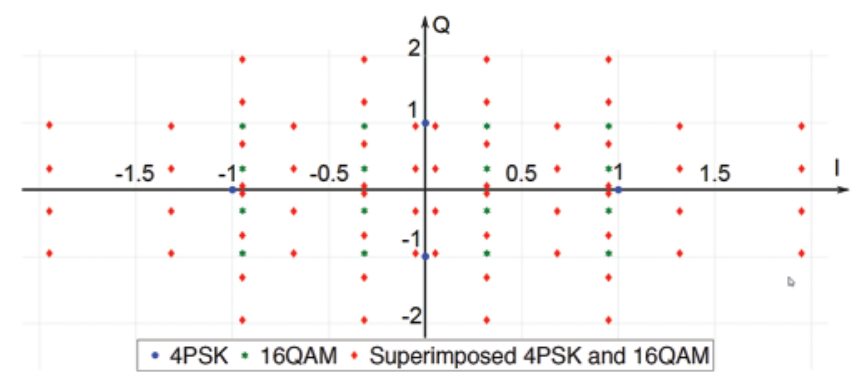

Figure 2: Constellations: 4PSK signal, 16QAM signal, and superimposed 4PSK and 16QAM signal

At $R$, the superimposed modulated useful information is affected by the noise and results a dispersion of the constellation points from their appropriate positions. Hence, at time instant $t$, we assume that the received signal $\mathbf{y}_{R, t}=\left(y_{R, t}^{(1)}, \ldots, y_{R, t}^{\left(N_{R}\right)}\right)^{T}$ is given by

$\mathbf{y}_{R, t}=\sqrt{P_{U_{1} R}} \mathbf{H}_{U_{1} R} \mathbf{x}_{1, t}+\sqrt{P_{U_{2} R}} \mathbf{H}_{U_{2} R} \mathbf{x}_{2, t}+\mathbf{n}_{R, t}$,

where $P_{U_{1} R}$ and $P_{U_{2} R}$ are the signal powers at $R$ from the users $U_{1}$ and $U_{2}$, respectively. $\mathbf{H}_{U_{i} R} \in$ $\mathbb{C}^{N_{R} \times N_{U_{i}}}$ represents the channel matrix between $U_{\mathrm{i}}$ and $R$. It is given by

$\mathbf{H}_{U_{i} R}=\left(\begin{array}{llll}h_{11} & h_{12} & \ldots & h_{1 N_{U_{i}}} \\ h_{21} & h_{22} & \ldots & h_{2 N_{U_{i}}} \\ \vdots & \vdots & \ldots & \vdots \\ h_{N_{R} 1} & h_{N_{R} 2} & \ldots & h_{N_{R} N_{U_{i}}}\end{array}\right)$. 
$\mathbf{H}_{U_{i} R}$ are modeled with Nakagami- $m_{i}$ fading, $i=1,2$. Here, the factor $m_{i}$ represents the Nakagami- $m$ severity parameter of the $U_{\mathrm{i}}-R$ channel. As the value of $m_{i}$ increases, the fading severity decreases. The case of $m_{i}=1$ corresponds to the Rayleigh fading. $\mathbf{x}_{1, t}$ and $\mathbf{x}_{2, t}$ are the modulated data vectors at $U_{1}$ and $U_{2}$, respectively. Finally, $\mathbf{n}_{R, t}$ is a circularly complex Gaussian noise of variance $\sigma_{n}^{2}$. The SNR average of the $r$ th relay antenna from the $j$ th antenna of user $U_{i}$ is expressed as

$\bar{\gamma}_{U_{i} 0}=\mathbb{E}\left[\gamma_{U_{i}, r j}\right]=\mathbb{E}\left[\frac{P_{U_{i}}}{\sigma_{n}^{2}}\left|h_{r j}\right|^{2}\right]$,

where $i \in\{1,2\}, r \in\left\{1, \ldots, N_{R}\right\}$ and $j \in\left\{1, \ldots, N_{U_{i}}\right\}$.

In the second time slot, a linear processing is performed on the received signal at the relay node $R$. In fact, a $\mathrm{ZF}$ processing is applied to the superimposed signals to eliminate the interpair and the inter-user interferences. The linear processing matrix, denoted by $\mathbf{P}_{L} \in \mathbb{C}^{2 N_{U} \times N_{R}}$, is given by

$\mathbf{P}_{L}=\left(\left[\mathbf{H}_{U_{1} R}, \mathbf{H}_{U_{2} R}\right]^{H}\left[\mathbf{H}_{U_{1} R}, \mathbf{H}_{U_{2} R}\right]\right)^{-1}\left[\mathbf{H}_{U_{1} R}, \mathbf{H}_{U_{2} R}\right]^{H}$.

Here, we suppose that the relay node $R$ has a perfect knowledge of $\mathbf{H}_{U_{1} R}$ and $\mathbf{H}_{U_{2} R}$ since the estimation of the backward channels can be performed based on pilot signaling [37]. Therefore, the transformed signal is written as

$\mathbf{z}_{R}=\mathbf{P}_{L} \mathbf{y}_{R, t}$.

In this work, we consider the distances between nodes. Given the fact that $d_{S D}$ denotes the Euclidean distance between a source, denoted by $S$ and a destination, denoted by $D$, the path loss between $S$ and $D$ is defined in [38] as

$g_{S D}=\frac{\varsigma}{d_{S D}^{\eta}}$

where $\varsigma$ represents a constant depending on both the environment and the carrier wavelength, and $\eta$ denotes the path-loss exponent and usually varies between two and six. Here, we consider that the two users $U_{1}$ and $U_{2}$ have the same transmission power $P_{U}=P_{U_{1}}=P_{U_{2}}$. To ensure a fair comparison with one-hop transmission, consider that $P_{U U^{\prime}}=P_{U_{1} U_{2}}=P_{U_{2} U_{1}}$ to be the power of the received signal at the end-node of the $U_{1} U_{2}$ link (i.e., direct link). Hence, the power of the received signal at $R$ from the user $U_{i}$, denoted by $P_{U_{i} R}$, can be written as

$P_{U_{i} R}=\frac{\varsigma}{d_{U_{i} R}^{\eta}} P_{U}=\left(\frac{d_{U_{1} U_{2}}}{d_{U_{i} R}}\right)^{\eta} P_{U U^{\prime}}$

where $d_{U_{1} U_{2}}$ denotes the distance between the user nodes $U_{1}$ and $U_{2}$, while $d_{U_{i} R}$ represents the distance between the user $U_{i}, i=1,2$ and the relay $R .\left(d_{U_{1} U_{2}} / d_{U_{i} R}\right)^{\eta}$ represents the $U_{i}-R$ power gain with respect to $P_{U U^{\prime}}$. This latter quotient is denoted by $G_{U_{i} R}, i=1,2$. The average SNRs of the $U_{i}-R$ link is written based on the normalized fading coefficients as

$\bar{\gamma}_{U_{i} R}=\frac{P_{U_{i} R}}{\sigma_{n}^{2}}=G_{U_{i} R} \frac{P_{U U^{\prime}}}{\sigma_{n}^{2}}, \quad i=1,2$. 
The quotient $P_{U U^{\prime}} / \sigma_{n}^{2}$ represents the reference SNR and we denote it by $\bar{\gamma}$.

In the second time slot, $R$ broadcasts the superimposed signal to the users with an additional overhead containing the estimated users modulations. A mandatory condition to obtain the correct information at both $U_{1}$ and $U_{2}$ is the appropriate detection of the modulations used by $U_{1}$ and $U_{2}$. Thus, $R$ should perfectly detect the users modulations from the equalized signal $\mathbf{z}_{R}$.

In this context, we propose an algorithm for classifying superimposed modulations. It is mainly composed of two subsystems. The first subsystem allows to extract the higher-order statistics (HOSs) features from the equalized signal $\mathbf{z}_{R}$, while the second subsystem allows detecting the users modulations pair based on the extracted features and the MultiBoostAB classifier.

In the following, we describe the proposed modulation classification algorithm.

\section{Proposed Superimposed Modulations Classification Algorithm}

The proposed superimposed modulations classification algorithm is divided into two main steps. The first one consists of extracting a set of appropriate features, while the second one concerns the classification based on supervised machine learning techniques. In the following, we explain these two steps.

\subsection{Extraction of Discriminating Features}

The higher-order statistics (HOSs) composed by the higher-order moments (HOMs) and the higher-order cumulants (HOCs) have shown in several recent existing works in literature their ability to classify modulations for MIMO systems [39]. In fact, each modulation scheme can be characterized by a set of HOMs and HOCs. The use of HOSs up to order eight allow the correct classification of various modulation types [40].

The $j^{\text {th }}$-order HOM of the equalized sequence at the $a^{\text {th }}$ antenna $\left(\mathbf{z}_{R}^{(a)}=\left(z_{R, 1}^{(a)}, \ldots, z_{R, N}^{(a)}\right)\right)$ is given by [41]

$M_{j k}\left(\mathbf{z}_{R}^{(a)}\right)=E\left[\left(\mathbf{z}_{R}^{(a)}\right)^{j-k}\left(\overline{\mathbf{z}_{R}^{(a)}}\right)^{k}\right], \quad a=1, \ldots, N_{R}$.

An estimation of the HOMs can be expressed as

$\hat{M}_{j k}\left(\mathbf{z}_{R}^{(a)}\right)=\frac{1}{N} \sum_{n=1}^{N}\left(z_{R, n}^{(a)}\right)^{j-k}\left(\overline{z_{R, n}^{(a)}}\right)^{k}$.

The $j^{\text {th }}$-order HOC of the $\mathbf{z}_{R}^{(a)}$ signal can be expressed as

$C_{j k}\left(\mathbf{z}_{R}^{(a)}\right)=\operatorname{Cum}[\underbrace{\mathbf{z}_{R}^{(a)}, \ldots, \mathbf{z}_{R}^{(a)}}_{(j-k) \text { times }}, \underbrace{\overline{z_{R}^{(a)}}, \ldots, \overline{z_{R}^{(a)}}}_{(k) \text { times }}]$.

The $j^{\text {th }}$-order HOC may be written as a function of lower and equal ordered HOMs as follows

$\operatorname{Cum}\left[\mathbf{z}_{R 1}^{(a)}, \ldots, \mathbf{z}_{R j}^{(a)}\right]=\sum_{\Psi}(-1)^{\delta-1}(\delta-1) ! \prod_{\varphi \in \Psi} E\left[\prod_{c \in \varphi} \mathbf{z}_{R c}^{(a)}\right]$, 
where $\Psi$ runs through the list of all partitions of $\{1, \ldots, j\}, \varphi$ runs through the list of all blocks of the partition $\Psi$ and $\delta$ is the elements number of the partition $\Psi$. We raise each HOC to the power $2 / j$ since the magnitude of HOCs increases with their order [42].

The process of classification of modulations pair for the received signal $y_{R, t}$ is illustrated in Fig. 3. A training phase is firstly launched to build a model using the MultiBoosting through the use of a learning database (LDB). Then, the test phase is done to classify the modulations pair of $y_{R, t}$ based on the model that is already built with the LDB. In the following, we describe the MultiBoosting classifier.

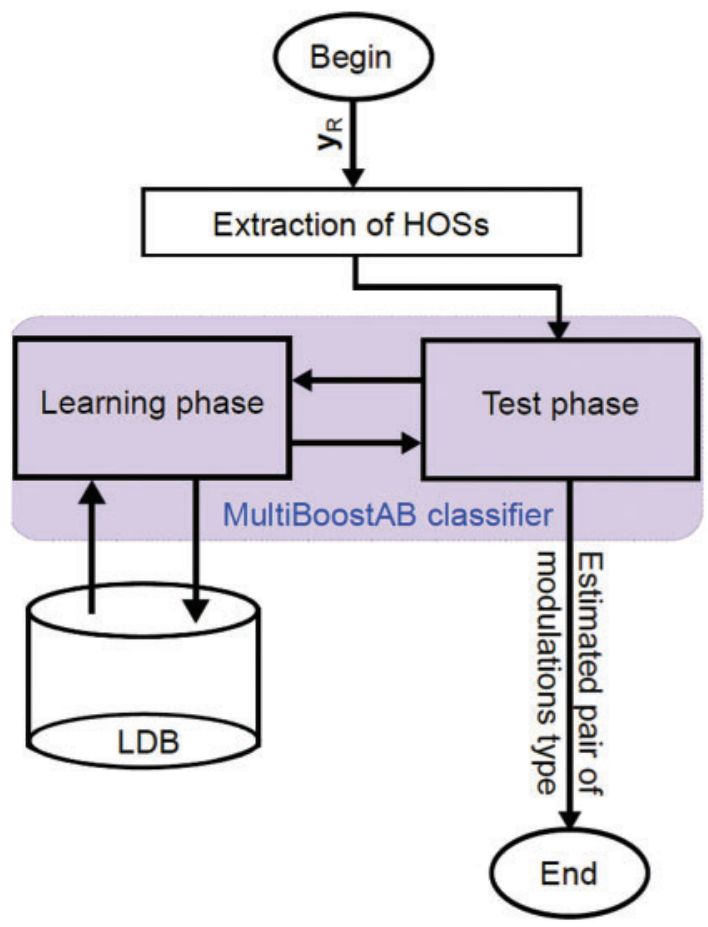

Figure 3: Process of classification of modulations pair for the received signal $\mathrm{y}_{\mathrm{R}, \mathrm{t}}$

\subsection{MultiBoostAB}

In this work, we use the MultiBoosting (MultiBoostAB) classifier, which is a combination of the Boosting and the Wagging techniques [43]. We present in the Algorithm 1 the pseudocode of MultiBoostAB classifier.

The idea is to harness the benefits provided by both techniques. In fact, this classifier takes advantage of Wagging's superior variance reduction in addition to the AdaBoost's high bias and variance reduction. Here, we employ the C4.5 (J48) [44] as a base learning algorithm since with this latter MultiBoost classifier provides a good prediction comparing to the AdaBoost classifier. To prove the effectiveness of MultiBoostAB operating with $\mathrm{J} 48$ classifier in superimposed modulation classification, we carry out a comparative study with the J48 classifier alone that outperforms the performance of multilayer perceptron classifier trained with resilient backpropagation training algorithm [45]. 


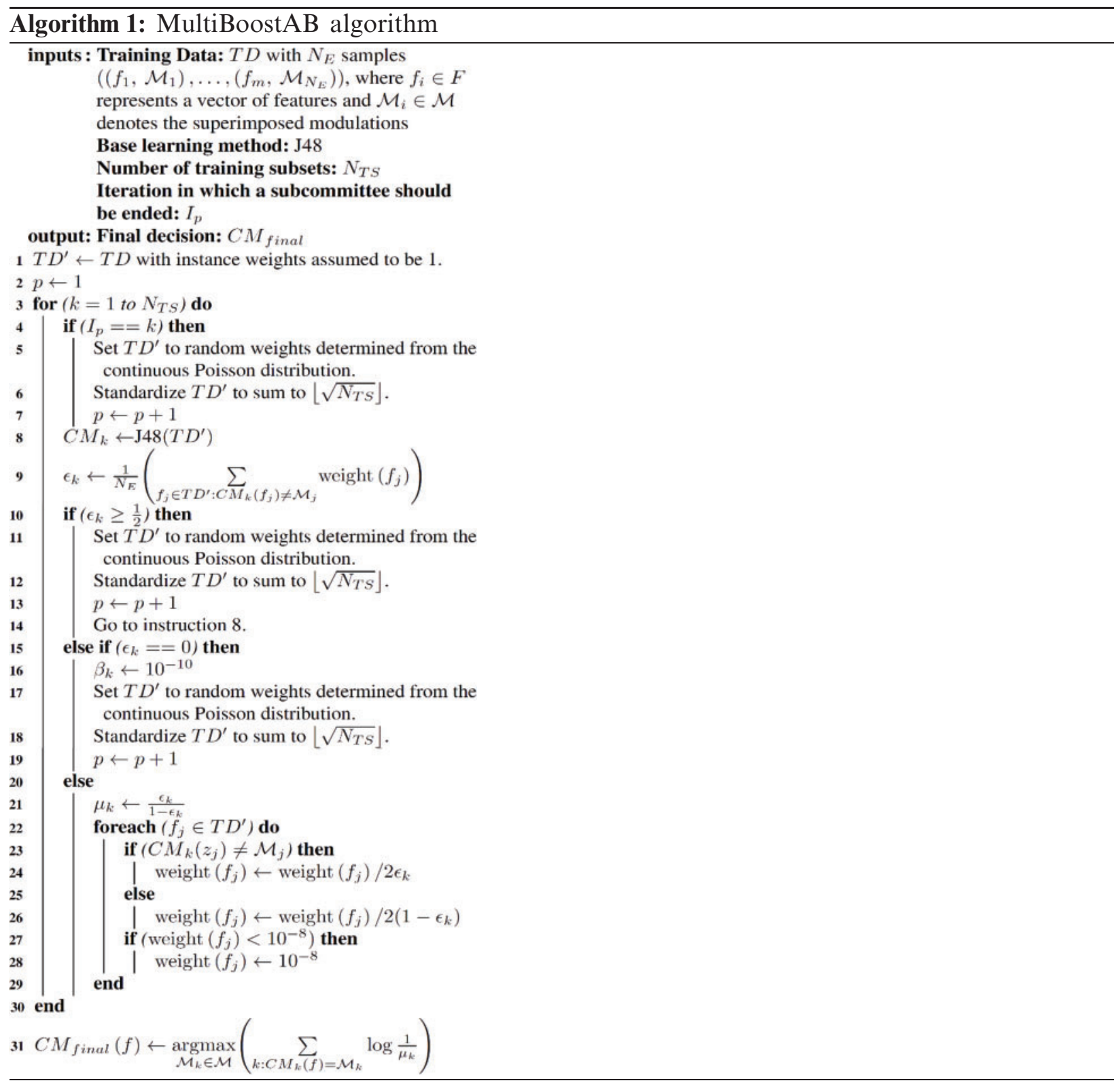

\subsection{Metrics Used for Performance Evaluation of Classifiers}

In this study, we compare between classifiers using true positive (TP) rate, false positive (FP) rate, precision, recall and F-Measure metrics. The precision, recall and F-measure are given respectively as

$$
\begin{aligned}
& \text { precision }=\frac{\mathrm{TP}}{\mathrm{TP}+\mathrm{FP}}, \\
& \text { recall }=\frac{\mathrm{TP}}{\mathrm{TP}+\mathrm{FN}}, \\
& \mathrm{F} \text {-measure }=2 \times \frac{\text { precision } \times \text { recall }}{\text { precision }+ \text { recall }} .
\end{aligned}
$$




\section{Simulation Results}

Simulation experiments are conducted to demonstrate the advantages of the proposed automatic classification modulation algorithm. Here, we apply our proposal to classify ten combinations of modulation pairs, i.e., $\mathcal{M}=\{(16 \mathrm{QAM}, 16 \mathrm{QAM}),(16 \mathrm{QAM}, 64 \mathrm{QAM}),(16 \mathrm{QAM}, 2 \mathrm{PSK})$, (16QAM, 4PSK), (64QAM, 64QAM), (64QAM, 2PSK), (64QAM, 4PSK), (2PSK, 2PSK), (2PSK, 4PSK), (4PSK, 4PSK)\}.

For each pair in $\mathcal{M}$, we construct a training set with 200 superimposed signals, where the user messages $\mathbf{x}_{1, t}$ and $\mathbf{x}_{2, t}$ of $U_{1}$ and $U_{2}$, respectively, are created in a random manner. For each signal, the number of symbols $N$ is fixed to 10000 symbols. Here, the all channels are subject to Nakagami $m$ fading. 1000 Monte Carlo trials are generated as a test set for each superimposed pair in $\mathcal{M}$. Thus, the test set contains 10000 Monte Carlo trials results (i.e., Num $_{\text {total }}=10 \times 1000$ ). We consider a free-space path loss model with $\eta=2$ and the number of antennas at each node is fixed to $N_{U}=4$. In the training and test phases, the set of features is building using the HOSs (HOCs and HOMs) of $\mathbf{z}_{R}^{(a)}$. Finally, we use MultiBoostAB with J48 as a base classifier, where the number of training subset $N_{T S}$ is equal to 10 [46]. This latter setting shows that the MultiBoostAB classifier provides a good compromise between the probability of modulation classification and the speed of the training phase.

In this work, the probability of the correct classification is computed by

$P=\frac{\sum_{\psi_{m} \in \mathcal{M}} N_{\psi_{m}}}{\text { Num }_{\text {total }}} \times 100$,

where $N_{\psi_{m}}$ is the number of trials for which the pair modulations $\psi_{m} \in \mathcal{M}$ is perfectly classified. For each test trial, a collaboration between all $N_{R}$ antennas is made in order to take the decision. Indeed, the pair of modulations having the majority of votes represents the estimated modulations pair.

\subsection{Accuracy of the MultiBoostAB Classifier}

We firstly evaluate the performance of the MultiBoostAB classifier using a 10-fold crossvalidation [47] on the training set described above. Tab. 2 displays the detailed accuracy by superimposed modulations. By analyzing the average of the TP rate, FP rate, precision, recall, F-Measure and receiver operating characteristic (ROC) area, it is clearly shown that the MultiBoost $\mathrm{AB}$ offers a good classification performance. In fact, the values of $\mathrm{TP}$ rate, precision, recall, F-Measure and ROC area are very close to 1 and the value of the FP rate is very close to 0 . Therefore, the MultiBoostAB classifier is efficient to automatically classify superimposed modulations.

\subsection{Impact of the Nakagami-m Fading Parameter}

Fig. 4 shows the impact of the channel fading severity on the $P$, for $\mathcal{M}$ and $d_{U_{1} R}=d_{U_{2} R}=$ 0.5. It is apparent that the probability of the classification decreases when decreasing fading parameter $m$ since the fading becomes more and more severe. We also observe that the proposed algorithm has the ability to classify superimposed modulations at low SNR even in the case where the fading severity parameter is set to 0.5 (i.e., worst-case). By comparing the performance of the MultiBoostAB with J48 classifier alone, it is clearly shown that the MultiBoostAB classifier offers a gain compared to $\mathrm{J} 48$ classifier in terms of superimposed modulations classification. For example, at $95 \%$ of superimposed modulations classification, the MultiBoostAB classifier provides 
a SNR gain of about $0.5 \mathrm{~dB}$ compared to J48 for the case where $m=0.5$. Consequently, the MultiBoostAB classifier is more appropriate in superimposed modulations classification.

Table 2: Detailed accuracy by superimposed modulations for the MultiBoostAB classifier with $N_{U}=4, N=10000, \eta=2, d_{U_{1} R}=d_{U_{2} R}=0.5, m=0.5$ and $\bar{\gamma}=5 \mathrm{~dB}$

\begin{tabular}{lllllll}
\hline $\mathcal{M}_{i}$ & TP rate & FP rate & Precision & Recall & F-measure & ROC area \\
\hline (16QAM, 16QAM) & 0.976 & 0.001 & 0.994 & 0.976 & 0.985 & 0.999 \\
$($ 16QAM, 64QAM) & 0.979 & 0.003 & 0.97 & 0.979 & 0.974 & 0.999 \\
(16QAM, 2PSK) & 0.999 & 0.001 & 0.994 & 0.999 & 0.996 & 1 \\
(16QAM, 4PSK) & 0.995 & 0 & 0.996 & 0.995 & 0.996 & 0.999 \\
(64QAM, 64QAM) & 0.991 & 0.002 & 0.984 & 0.991 & 0.988 & 1 \\
(64QAM, 2PSK) & 0.994 & 0 & 0.997 & 0.994 & 0.996 & 1 \\
(64QAM, 4PSK) & 0.996 & 0 & 0.996 & 0.996 & 0.996 & 0.998 \\
(2PSK 2PSK) & 1 & 0 & 0.999 & 1 & 0.999 & 1 \\
(2PSK, 4PSK) & 0.998 & 0 & 0.999 & 0.998 & 0.998 & 0.999 \\
(4PSK, 4PSK) & 1 & 0 & 0.999 & 1 & 0.999 & 1 \\
Average & 0.993 & 0.001 & 0.993 & 0.993 & 0.993 & 0.999 \\
\hline
\end{tabular}

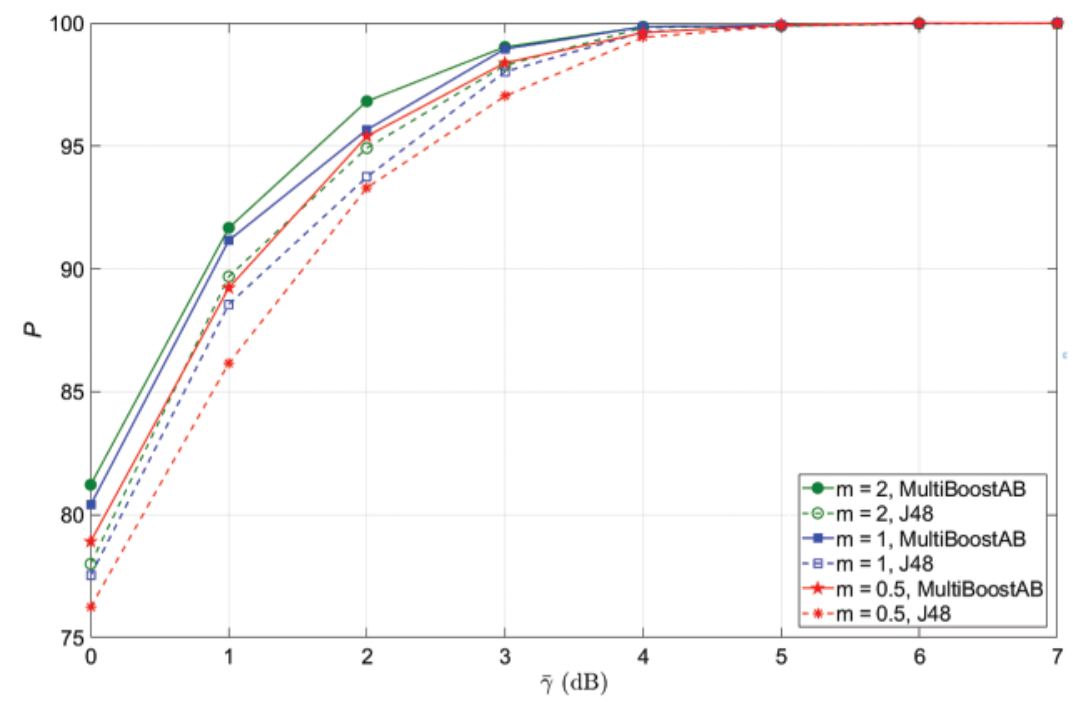

Figure 4: Impact of the Nakagami parameter, $m=\{0.5,1,2\}$, on the average probability of correct classification, $P$, for $\mathcal{M}, N_{U}=4, N=10000, \eta=2$ and $d_{U_{1} R}=d_{U_{2} R}=0.5$ using MultiBoostAB and $\mathrm{J} 48$ classifiers

\subsection{Impact of the Symbols Number}

Fig. 5 presents the average probability of correct classification, $P$, as a function of $\bar{\gamma}$, for many values of symbols number $N$ using MultiBoostAB classifier. The increase of $N$ leads 
to an improvement in $P$ since the HOMs estimation accuracy calculated in (8) increases when increasing $N$.

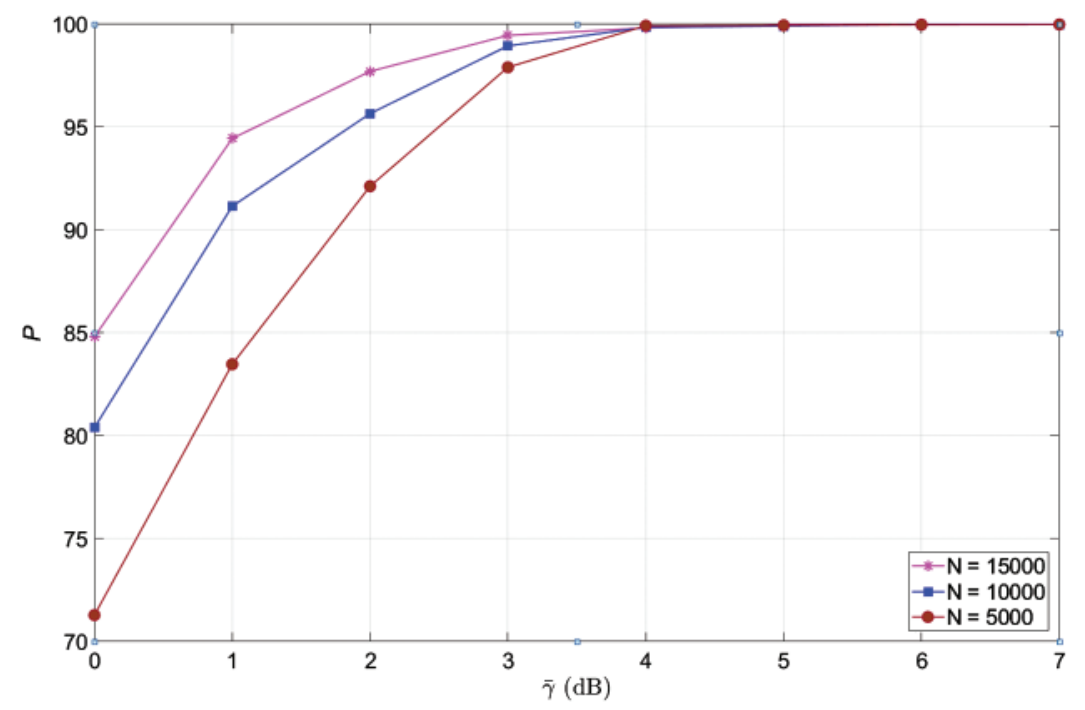

Figure 5: Impact of the symbols number, $N$, on the average probability of correct classification, $P$, for $\mathcal{M}, N_{U}=4, m=0.5, \eta=2$ and $d_{U_{1} R}=d_{U_{2} R}=0.5$ using MultiBoostAB classifier

\subsection{Impact of the Relay Position}

As seen in Eq. (6), we can incorporate different relay positions, where all distances involved in the calculation of the power gain are relative to the distance between the two users $U_{1}$ and $U_{2}$. In fact, we assume that $d_{U_{1} R}+d_{U_{2} R}=1$.

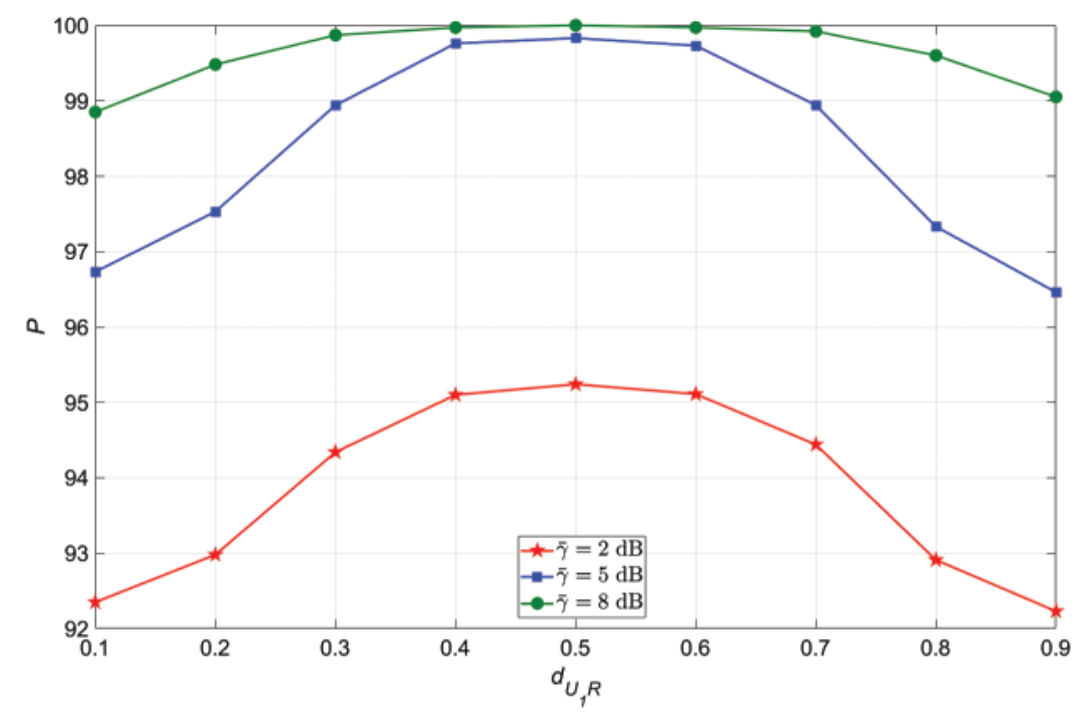

Figure 6: Impact of the distance between $U_{1}$ and $R, d_{U_{1} R}$, on the average probability of correct classification, $P$, for $\mathcal{M}, N_{U_{1}}=N_{U_{2}}=N_{R}=4, N=10000, \eta=2, m=0.5$ and $d_{U_{1} U_{2}}=d_{U_{1} R}+$ $d_{U_{2} R}=1$ 
Fig. 6 illustrates the average probability of correct classification of the proposed algorithm, $P$, as a function of $\bar{\gamma}$ at different relay positions for $\mathcal{M}$. It is clearly shown that, when the relay is located exactly in the middle (i.e., $d_{U_{1} R}=d_{U_{1} R}=0.5$ ), the best performance is obtained. For example, a good performance $(P \cong 100 \%)$ is reached at $d_{U_{1} R}=0.5$, while only $97 \%$ is achieved at $d_{U_{1} R}=0.1$ for $\bar{\gamma}=5 \mathrm{~dB}$. It can be also shown from this Figure that the proposed algorithm achieves an excellent performance $(P \cong 100 \%)$ at all relay positions and for a low SNR $(\bar{\gamma}=8 \mathrm{~dB})$.

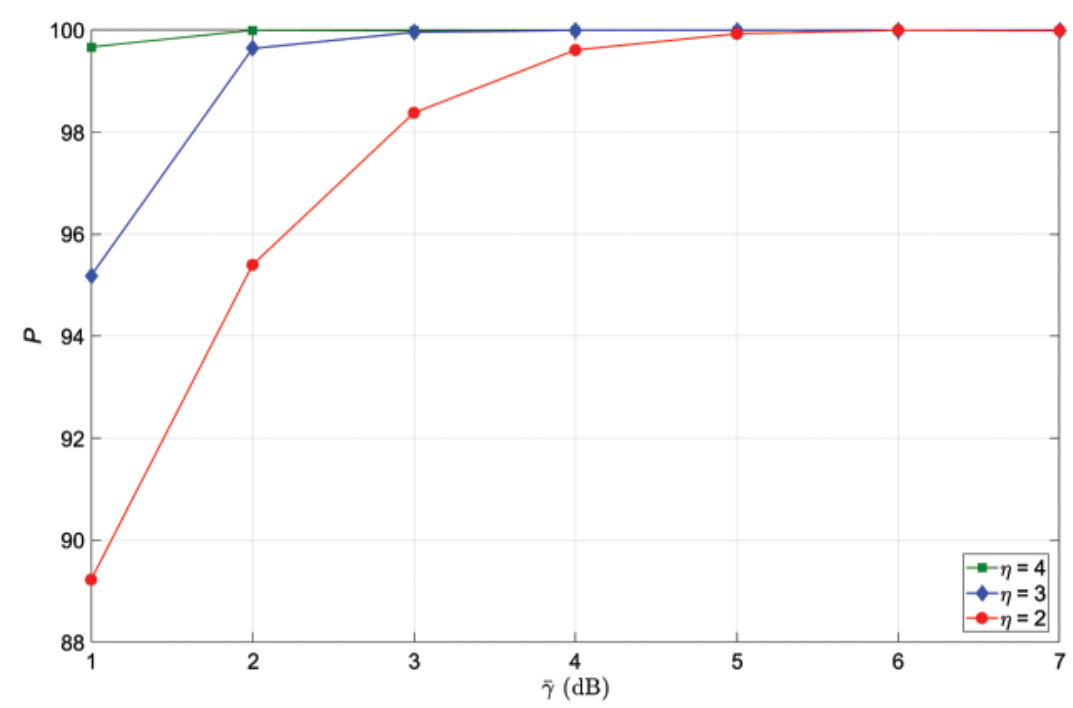

Figure 7: Impact of the path loss exponent, $\eta$, on the average probability of correct classification, $P$, for $\mathcal{M}, N_{U_{1}}=N_{U_{2}}=N_{R}=4, N=10000, m=0.5$ and $d_{U_{1} R}=d_{U_{2} R}=0.5$

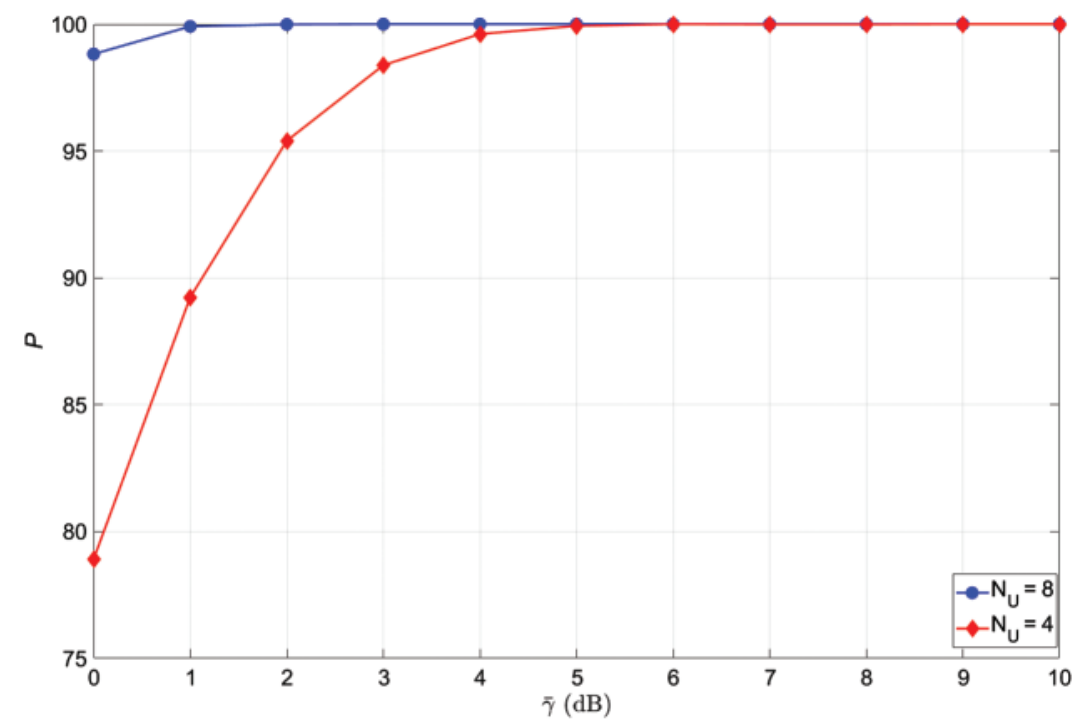

Figure 8: Impact of the antennas number, $N_{U}=N_{U_{1}}=N_{U_{2}}=N_{R}$, on the average probability of correct classification, $P$, for $\mathcal{M}, \eta=2, N=10000, m=0.5$ and $d_{U_{1} R}=d_{U_{2} R}=0.5$ 


\subsection{Impact of the Path Loss Exponent}

Fig. 7 presents the average probability of correct classification of the proposed algorithm, $P$, as a function of $\bar{\gamma}$, for various values of $\eta$, using $\mathcal{M}$ and $d_{U_{1} R}=d_{U_{2} R}=0.5$. The increase of $\eta$ leads to a considerable improvements in $P$. Here, the gain is obtained thanks to the increase of the $\bar{\gamma}$ at $R$ when increasing $\eta$ for the same reference $\bar{\gamma}$ as seen in Eq. (6).

\subsection{Impact of the Antenna Number}

Fig. 8 shows the average probability of correct classification of the proposed algorithm, $P$, as a function of $\bar{\gamma}$, for $N_{U}=N_{U_{1}}=N_{U_{2}}=N_{R}=\{4,8\}$, using $\mathcal{M}$ and $d_{U_{1} R}=d_{U_{2} R}=0.5$. One can see that $P$ is significantly increased when setting $N_{U}$ to 8 . In fact, a good performance is achieved at $\bar{\gamma}=1 \mathrm{~dB}$ for $N_{U}=8$, whereas the same performance is obtained at $\bar{\gamma}=5 \mathrm{~dB}$ for $N_{U}=4$.

\section{Conclusion and Future Work}

We have proposed an automatic classification algorithm of superimposed modulations designed for MIMO TWCR network over Nakagami- $m$ channels. At the relay node, we have extracted a set of HOSs from the superimposed received symbols as features extraction. Then, we have employed the MultiBoostAB classifier. Simulations were performed to show the performance of the proposed classification modulation algorithm based on several metrics. We have demonstrated that our proposal has the ability to provide good performances at a low SNR in the case where the fading severity parameter is set to 0.5 (i.e., worst-case). We have also carried out a comparative study between our proposal using MultiBoostAB classifier with J48 classifier. Through simulation results, we have clearly showed that the performance of MultiBoostAB on the superimposed modulations classifications outperforms the one of J48 classifier. Finally, we have studied the impact of the symbols number, path loss exponent and relay position on the performance of the proposed automatic classification superimposed modulations in terms of probability of correct classification.

In the future work, we will investigate the use of deep learning based neural networks in order to further improve the probability of the correct classification of superimposed modulations at low SNR values.

Funding Statement: This work was supported by Jouf University.

Conflicts of Interest: The authors declare that they have no conflicts of interest to report regarding the present.

\section{References}

[1] L. F. Xie, I. W.-H. Ho, Z. H. Situ, L. Lu and W. Lu, "Channel-coded physical-layer network coding with OFDM modulation," IEEE Access, vol. 6, pp. 22267-22280, 2018.

[2] Z. Xie, P. Chen, Z. Mei, S. Long, K. Cai et al., "Polar-coded physical layer network coding over twoway relay channels," IEEE Communications Letters, vol. 23, no. 8, pp. 1301-1305, 2019.

[3] T. Peng, Y. Wang, A. G. Burr and M. R. Shikh-Bahaei, "Physical layer network coding in network MIMO: A new design for 5G and beyond," IEEE Transactions on Communications, vol. 67, no. 3, pp. 2024-2035, 2018.

[4] F. Jamil, A. Javaid, T. Umer and M. H. Rehmani, "A comprehensive survey of network coding in vehicular ad-hoc networks," Wireless Networks, vol. 23, no. 8, pp. 2395-2414, 2017. 
[5] L. F. Xie, I. W.-H. Ho, S. C. Liew, L. Lu and F. C. Lau, "The feasibility of mobile physical-layer network coding with BPSK modulation," IEEE Transactions on Vehicular Technology, vol. 66, no. 5, pp. 3976-3990, 2016.

[6] S. Chaoui, H. Ben Chikha and A. Masmoudi, "Network-coded SIR-based distributed coding scheme: A new soft estimate modelling and performance analysis," AEU-International Journal of Electronics and Communications, vol. 70, no. 6, pp. 822-832, 2016.

[7] S. Zhang, S. C. Liew and P. P. Lam, "Hot topic: Physical-layer network coding," in Proc. 12th Annual ACM Int. Conf. on Mobile Computing and Networking, New York, NY, USA, pp. 358-365, 2006.

[8] W. Nam, S.-Y. Chung and Y. H. Lee, "Capacity of the Gaussian two-way relay channel to within 1/2 bit," IEEE Transactions on Information Theory, vol. 56, no. 11, pp. 5488-5494, 2010.

[9] T. Huang, T. Yang, J. Yuan and I. Land, "Design of irregular repeat-accumulate coded physical-layer network coding for Gaussian two-way relay channels," IEEE Transactions on Communications, vol. 61, no. 3, pp. 897-909, 2013.

[10] H. Zhang and L. Cai, "Design of channel coded heterogeneous modulation physical layer network coding," IEEE Transactions on Vehicular Technology, vol. 67, no. 3, pp. 2219-2230, 2017.

[11] I. Parvez, A. Rahmati, I. Guvenc, A. I. Sarwat and H. Dai, "A survey on low latency towards 5G: RAN, core network and caching solutions," IEEE Communications Surveys \& Tutorials, vol. 20, no. 4, pp. 3098-3130, 2018.

[12] H. Li, X.-W. Chang, Y. Cai and B. Champagne, "Efficient detection scheme for physical-layer network coding in multiway relay channels," IEEE Access, vol. 7, pp. 167639-167652, 2019.

[13] R. Y. Chang, S.-J. Lin and W.-H. Chung, "Symbol and bit mapping optimization for physical-layer network coding with pulse amplitude modulation," IEEE Transactions on Wireless Communications, vol. 12, no. 8, pp. 3956-3967, 2013.

[14] M. Noori and M. Ardakani, "On symbol mapping for binary physical-layer network coding with PSK modulation," IEEE Transactions on Wireless Communications, vol. 11, no. 1, pp. 21-26, 2011.

[15] P. Chen, L. Shi, S. C. Liew, Y. Fang and K. Cai, "Channel decoding for nonbinary physical-layer network coding in two-way relay systems," IEEE Transactions on Vehicular Technology, vol. 68, no. 1, pp. 628-640, 2018.

[16] X. Hu, Z. Chen and F. Yin, "Channel and delay estimation for asynchronous physical layer network coding," AEU-International Journal of Electronics and Communications, vol. 87, no. 12, pp. 101-106, 2018.

[17] X. Dang, Z. Huang, Q. Li and X. Yu, "Estimation of symbol timing in physical-layer network coding with arrival time differences," IEEE Communications Letters, vol. 21, no. 2, pp. 330-333, 2016.

[18] Q. Yang and S. C. Liew, "Asynchronous convolutional-coded physical layer network coding," IEEE Transactions on Wireless Communications, vol. 14, no. 3, pp. 1380-1395, 2014.

[19] Y. Huang, S. Wang, Q. Song, L. Guo and A. Jamalipour, "Synchronous physical-layer network coding: A feasibility study," IEEE Transactions on Wireless Communications, vol. 12, no. 8, pp. 4048-4057, 2013.

[20] L. Lu and S. C. Liew, "Asynchronous physical-layer network coding," IEEE Transactions on Wireless Communications, vol. 11, no. 2, pp. 819-831, 2011.

[21] Q. Nadeem, A. Kammoun and M. S. Alouini, "Elevation beamforming with full dimension MIMO architectures in 5G systems: A tutorial," IEEE Communications Surveys \& Tutorials, vol. 21, no. 4, pp. 3238-3273, 2019.

[22] M. Turan, M. Öner and H. A. Çırpan, "Joint modulation classification and antenna number detection for MIMO systems," IEEE Communications Letters, vol. 20, no. 1, pp. 193-196, 2015.

[23] M. Mohammadkarimi, E. Karami and O. A. Dobre, "A novel algorithm for blind detection of the number of transmit antenna," in Proc. Conf. on Cognitive Radio Oriented Wireless Networks, Doha, Qatar, pp. $441-450,2015$.

[24] M. R. Oularbi, S. Gazor, A. Aissa-El-Bey and S. Houcke, "Enumeration of base station antennas in a cognitive receiver by exploiting pilot patterns," IEEE Communications Letters, vol. 17, no. 1, pp. 8-11, 2012. 
[25] M. Marey, O. A. Dobre and B. Liao, "Classification of STBC systems over frequency-selective channels," IEEE Transactions on Vehicular Technology, vol. 64, no. 5, pp. 2159-2164, 2014.

[26] E. Karami and O. A. Dobre, "Identification of SM-OFDM and AL-OFDM signals based on their second-order cyclostationarity," IEEE Transactions on Vehicular Technology, vol. 64, no. 3, pp. 942-953, 2014.

[27] K. Liu, J. P. C. CDa Costa, H. C. So and A. L. De Almeida, "Semi-blind receivers for joint symbol and channel estimation in space-time-frequency MIMO-OFDM systems," IEEE Transactions on Signal Processing, vol. 61, no. 21, pp. 5444-5457, 2013.

[28] S. Cai, T. Matsumoto and K. Yang, "SIMO channel estimation using space-time signal subspace projection and soft information," IEEE Transactions on Signal Processing, vol. 60, no. 8, pp. 4219-4235, 2012.

[29] H. Ben Chikha, A. Almadhor and W. Khalid, "Machine learning for 5G MIMO modulation detection," Sensors, vol. 21, no. 5, pp. 1556, 2021.

[30] S. Zheng, P. Qi, S. Chen and X. Yang, "Fusion methods for CNN-based automatic modulation classification," IEEE Access, vol. 7, pp. 66496-66504, 2019.

[31] W. Ben Chikha and R. Attia, "On the utility of MIMO multi-relay networks for modulation identification over spatially-correlated channels," Telecommunication Systems, vol. 64, no. 4, pp. 735-747, 2017.

[32] F. Wang and X. Wang, "Fast and robust modulation classification via Kolmogorov-Smirnov test," IEEE Transactions on Communications, vol. 58, no. 8, pp. 2324-2332, 2010.

[33] J. Zhang, D. Cabric, F. Wang and Z. Zhong, "Cooperative modulation classification for multipath fading channels via expectation-maximization," IEEE Transactions on Wireless Communications, vol. 16, no. 10, pp. 6698-6711, 2017.

[34] F. Wang, O. A. Dobre, C. Chan and J. Zhang, "Fold-based Kolmogorov-Smirnov modulation classifier," IEEE Signal Processing Letters, vol. 23, no. 7, pp. 1003-1007, 2016.

[35] W. Ben Chikha, S. Chaoui and R. Attia, "Performance of Adaboost classifier in recognition of superposed modulations for MIMO TWRC with physical-layer network coding," in Proc. 25th Int. Conf. on Software, Telecommunications and Computer Networks, Split, Croatia, IEEE, pp. 1-5, 2017.

[36] W. Ben Chikha, S. Chaoui and R. Attia, "Identification of superposed modulations for two-way relaying MIMO systems with physical-layer network coding," IET Communications, vol. 11, no. 2, pp. 225-231, 2017.

[37] B. Zhang, Z. He, K. Niu and L. Zhang, "Robust linear beamforming for MIMO relay broadcast channel with limited feedback," IEEE Signal Processing Letters, vol. 17, no. 2, pp. 209-212, 2009.

[38] H. Ochiai, P. Mitran and V. Tarokh, "Design and analysis of collaborative diversity protocols for wireless sensor networks," in Proc. 60th IEEE Vehicular Technology Conf., Los Angeles, CA, USA, vol. 7, pp. 4645-4649, 2004.

[39] H. Ben Chikha, "Performance of Adaboost in modulation recognition for spatially-correlated cooperative MIMO systems," in Proc. IEEE Wireless Communications and Networking Conf., Barcelona, Spain, pp. 1-6, 2018.

[40] O. A. Dobre, A. Abdi, Y. Bar-Ness and W. Su, "Survey of automatic modulation classification techniques: Classical approaches and new trends," IET Communications, vol. 1, no. 2, pp. 137-156, 2007.

[41] P. McCullagh, Tensor Methods in Statistics. London, UK: Chapman Hall, 1987.

[42] C. M. Spooner, "On the utility of sixth-order cyclic cumulants for RF signal classification," in Proc. Conf. Record of Thirty-Fifth Asilomar Conf. on Signals, Systems and Computers, Pacific Grove, CA, USA, vol. 1, pp. 890-897, 2001.

[43] G. I. Webb, "Multiboosting: A technique for combining boosting and wagging," Machine Learning, vol. 40, no. 2, pp. 159-196, 2000.

[44] L. Rokach and O. Maimon, "Decision trees," in Data Mining and Knowledge Discovery Handbook. Boston, MA: Springer, pp. 165-192, 2005. 
[45] W. Ben Chikha, I. Dayoub, W. Hamouda and R. Attia, "Modulation recognition for MIMO relaying broadcast channels with direct link," IEEE Wireless Communications Letters, vol. 3, no. 1, pp. 50-53, 2014.

[46] M. Hall, E. Frank, G. Holmes, B. Pfahringer, P. Reutemann et al., "The WEKA data mining software: An update," ACM SIGKDD Explorations Newsletter, vol. 11, no. 1, pp. 10-18, 2009.

[47] P. Refaeilzadeh, L. Tang and H. Liu, "Cross-validation," Encyclopedia of Database Systems, vol. 5, pp. 532-538, 2009. 